\title{
Efficacy of perforator CTA of deep inferior epigastric artery perforator flap in preoperative planning of breast reconstruction: comparative study with CDUS
}

\author{
Hazem Hamed Soliman $^{1 *}\left(\mathbb{D}\right.$, Mohamed F. Abozeid ${ }^{2}$ and Maged Abdelrahman Moustafa ${ }^{1}$
}

\begin{abstract}
Background: The aim of the study is to compare the efficacy of perforator computed tomographic angiography (P-CTA) and color Doppler ultrasonography (CDUS) in preoperative planning of breast reconstruction with deep inferior epigastric artery perforator (DIEAP) flaps. From October 2018 to August 2019, 42 patients performed breast reconstruction with DIEAP flap procedures. All cases performed CDUS and multidetector perforator CT angiography (P-CTA) within 1 week prior to reconstructive surgery. The CDUS and P-CTA images were interpreted by two experienced vascular radiologists with 8- and 10-year experience, respectively, and compared with the surgical results.

Results: There were statistically significant differences $(P=0.0025)$ between CDUS and P-CTA in the detection of the number of perforators with P-CTA being more accurate than CDUS. There were significant differences between the P-CTA and surgical findings in measuring the caliber of dominant perforators $(P=0.002)$. CDUS was more accurate than P-CTA in this parameter showing sensitivity and specificity of 94 and $96 \%$, respectively. There were significant differences between CDUS and surgical findings in the detection of the course of the intramuscular segment of the perforator $(P=0.0028)$. P-CTA was more accurate than CDUS in this parameter showing sensitivity and specificity of 96 and $97 \%$, respectively.

Conclusion: The pre-operative mapping of perforators by CDUS and P-CTA proved to be effective and complementary to each other in the localization and vascular mapping of deep inferior epigastric artery perforators, thus greatly helping vascular surgeons in performing this surgical procedure.
\end{abstract}

Keywords: Inferior epigastric, Perforator, Computed tomographic angiography, CDUS

\footnotetext{
* Correspondence: hsoliman79@yahoo.com

'Diagnostic and Intervention Radiology Department, Cairo University

Hospitals, Kasr Al-Ainy, El-Manial, Cairo 11956, Egypt

Full list of author information is available at the end of the article
}

\section{Springer Open}

๑ The Author(s). 2020 Open Access This article is licensed under a Creative Commons Attribution 4.0 International License, which permits use, sharing, adaptation, distribution and reproduction in any medium or format, as long as you give appropriate credit to the original author(s) and the source, provide a link to the Creative Commons licence, and indicate if changes were made. The images or other third party material in this article are included in the article's Creative Commons licence, unless indicated otherwise in a credit line to the material. If material is not included in the article's Creative Commons licence and your intended use is not permitted by statutory regulation or exceeds the permitted use, you will need to obtain permission directly from the copyright holder. To view a copy of this licence, visit http://creativecommons.org/licenses/by/4.0/. 


\section{Background}

Breast cancer is the most common malignancy in women in Egypt accounting for approximately $38.8 \%$ of all malignancies in females [1].

Breast reconstructive surgery is usually performed by women who perform mastectomy as a definitive treatment for breast cancer or as a prophylactic procedure in cases with a high risk of breast cancer in order to preserve the aesthetic appearance of the breast [2].

Breast reconstruction can either be performed with artificial tissue such as saline or silicone implants or by utilizing autologous tissue such as abdominal tissue flaps. The latter yields better results with fewer incidences of complications [2].

The transverse rectus abdominis myocutaneous pedicle flap (TRAM) was the most commonly used autologous breast reconstruction technique in the past 40 years. However, it presented multiple complications including abdominal wall weakness, asymmetry, and herniation [3].

In recent years, a new autologous breast reconstructive technique emerged to overcome the drawbacks of the TRAM procedure called deep inferior epigastric artery perforator (DIEAP) flap. This is a microsurgical procedure that uses abdominal skin and fatty tissue vascularized flap to reconstruct the breast after mastectomy. This technique preserves the rectus abdominis muscle and fascia, thus reducing donor site morbidity and improving recovery period and healing resulting in a reconstructed breast mound with more aesthetic appealing characteristics [4].

Nevertheless, the DIEAP flap procedure is a technically challenging and time-consuming breast reconstructive technique. This is due to high anatomical variations of the anterior abdominal wall vascular supply between different individuals or even between each abdominal side of the same person. Therefore, pre-operative localization of the site, caliber, and intramuscular course and site of exit through the rectus abdominis fascia of the dominant perforating vessels is of utmost importance for the surgeon to obtain more favorable results with less post-operative complications [5].

To overcome these difficulties, several pre-operative radiological methods were used for the selection of the dominant perforators used to perfuse the DIEAP flap based on the site, caliber, and course of each perforator. The commonest radiological methods used are handheld acoustic Doppler sonography (ADS), color Doppler ultrasonography (CDUS), and multidetector computed tomography (MDCT) [6].

ADS is a non-invasive, portable, low cost, procedure with a relatively short time of acquisition. This explains the persistent intra-operative widespread use of ADS nowadays. On the other hand, ADS is operator dependent with low reproducibility lacking both hemodynamic information and image visualization which results in producing relatively high false-positive results [7].

Color Doppler ultrasonography is considered as the standard pre-operative examination for localization of perforators in DIEAP flap procedure. However, it has several disadvantages; being time-consuming, less reproducible, and its inability to produce the entire structural information required in a single-image accessible to the surgeon [7].

Perforator computed tomographic angiography (PCTA) with MDCT provides accurate information about the location, course, and caliber of the perforator with the ability to represent these findings in reconstructed multiplanar (MPR), maximum intensity projection (MIP), and volume-rendered (VR) reformats. On the other hand, the main disadvantages of the P-CTA are exposure to ionizing radiation and nephrotoxic contrast agent [8].

The objective of this study is to compare the efficacy of perforator computed tomographic angiography (PCTA) and color Doppler ultrasonography (CDUS) in preoperative planning of breast reconstruction with DIEAP flaps.

\section{Methods \\ Study population and design}

From October 2018 to August 2019, 42 patients performed breast reconstruction with DIEAP flap procedures. The age ranged between 27 years and 63 years (mean age 41.5). The patients were referred from the plastic surgery department, and the study was approved by the ethics committee of our institute. All patients included in this research gave written informed consent to participate in this study. All cases performed CDUS and multidetector perforator CT angiography (P-CTA) within 1 week prior to reconstructive surgery. The CDUS and P-CTA images were interpreted by two experienced vascular radiologists with 8 and 10 years of experience, respectively, and compared with the surgical results.

- Inclusion criteria: Patients over 20 years with a history of mastectomy who are clinical candidates for breast reconstruction with deep inferior epigastric artery perforator flap procedure

- Exclusion criteria: Color Doppler ultrasound: No exclusion criteria P-CTA: allergy to iodinated contrast medium and serum creatinine level more than $1.6 \mathrm{mg} / \mathrm{dl}$

\section{Color Doppler ultrasound procedure}

1) Doppler ultrasound device was utilized (Elegra; Siemens Medical Systems, Erlangen, Germany) with 
linear array transducers $(5-10 \mathrm{MHz})$ while curved and phased array transducers were used for obese patients.

2) The patient was examined in a supine position using enough gel to facilitate visualization.

3) Each deep inferior epigastric artery was evaluated on either side of the abdomen in an area corresponding to the flap dimensions; extending 2 $\mathrm{cm}$ above the level of the umbilicus cranially, down to the inguinal ligaments caudally, and $10 \mathrm{~cm}$ laterally. Longitudinal and axial scanning was performed to identify the main perforators below the umbilicus on either side (Fig. 1).

4) The following measurements were recorded to detect the dominant perforators on each side; the calibers of the perforating arteries and veins, the length and course of the intramuscular segment of the perforators, and the site of emergence of the perforators through the rectus abdominis fascia which was marked on the skin and recorded in relation to the umbilicus (considered as the reference zero point).

\section{Perforator CT angiography protocol}

1) The examination was done with a sixty-four multidetector CT scanner (TOSHIBA Medical Systems, Aquillion, Japan) using the following parameters; 400-msec gantry rotation, $120-\mathrm{kV}$ tube voltage, and 300-mA tube current.
2) The patient was scanned in the supine position in the caudo-cranial direction from the femoral trochanteric region to $5 \mathrm{~cm}$ above the umbilicus.

3) A $90 \mathrm{ml}$ of nonionic iodinated contrast medium with concentration of $350 \mathrm{mg}$ of iodine per milliliter was injected through an 18 gauge intravenous catheter at the antecubital vein using an automatic double head injector at a rate of $4 \mathrm{ml} / \mathrm{s}$ followed by a 50-ml saline flush with the same flow rate. The region of interest (ROI) was placed at the common femoral artery.

4) The obtained images were reconstructed at a slice width of $0.8 \mathrm{~mm}$ and an interval of $0.5 \mathrm{~mm}$. A postprocessing workstation (Vitrea version 3.0.1, Vital Images, Plymouth, MN, USA) was used for interpretation. Two-dimensional (2D) image reconstructions, such as maximum intensity projection (MIP), multi-planar reformation (MPR), and 3D volume rendering (VR) techniques (Fig. 2), were used interchangeably to complete the evaluation.

5) The following parameters were recorded to detect the dominant perforators; the site and caliber of each perforator as it pierces the rectus abdominis muscle fascia was demonstrated on the axial and sagittal thin maximum intensity projection (MIP) images. The length and course of the intramuscular segment of each perforator were assessed on the multiplanar and thin MIP reconstructed images (Fig. 3). The exact location of each perforator in relation to the umbilicus (considered as the
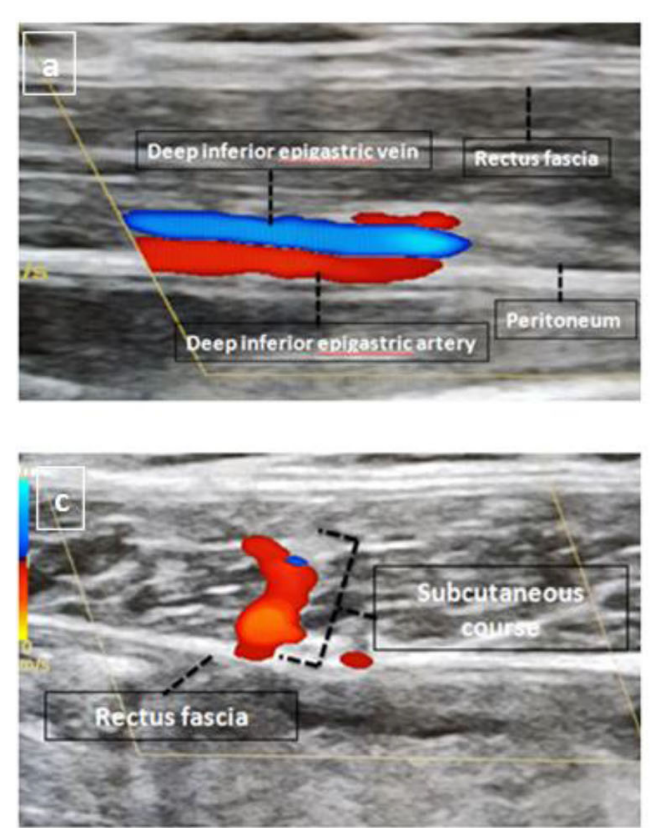

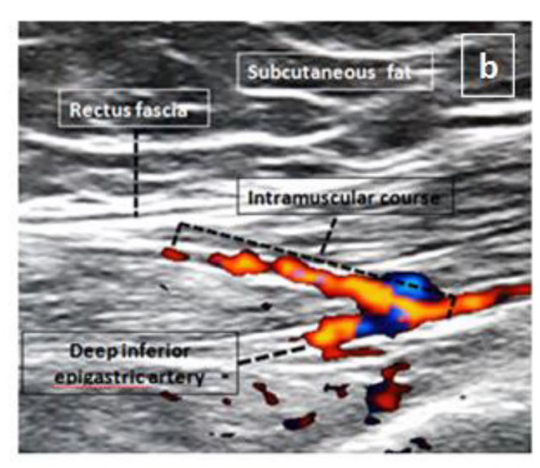

courses of a dominant perforator as it pierces the rectus fascia 

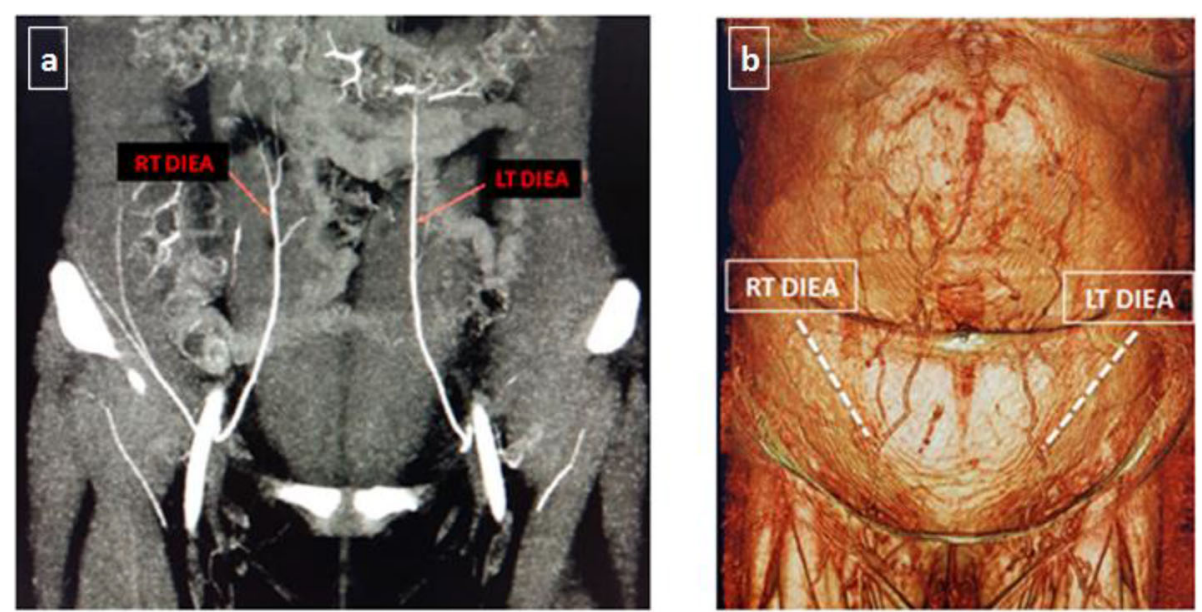

Fig. 2 Coronal image of the anterior abdominal wall showing maximum intensity projection (MIP) (a) and volume rendering (VR) reformat (b) of both deep inferior epigastric arteries

reference zero point) was plotted on a volumerendered (VR) image of the abdominal wall to facilitate surgical guidance (Fig. 4). Finally, the branching pattern of each deep inferior epigastric artery was identified based on the Moon and Taylor classification (Fig. 5).

\section{Intra-operative perforator flap methodology}

The patient lied in the supine position on the operating table. The abdomen and thigh regions were sterilized, and surgical drapes were placed over the surgical area from the chest to the mid-thigh region. Each deep inferior epigastric artery was exposed separately. An elliptical incision through the lower abdominal adipocutaneous tissue was performed with mobilization followed by dissection of the anterior rectus sheath and vertical incision of the rectus abdominus muscle fibers to delineate the origins of the perforators from the deep inferior epigastric artery. The dominant perforators were then identified. The mobilized flap was then placed over the breast recipient site followed by anastomosis of the perforator with the ipsilateral internal mammary artery (Fig. 6).

\section{Selecting perforating vessels for free flap breast reconstruction}

Based on the consultation with our surgical team, the key criteria for the selection of a dominant perforator were agreed upon as follows:

1) Site was identified on the volume-rendered images. The perforator must be infra-umbilical in location and located in close proximity to the umbilicus, i.e., less than $3 \mathrm{~cm}$ inferior and $3 \mathrm{~cm}$ lateral to the umbilicus.

2) Caliber was measured on the MIP images as the perforator pierces the anterior rectus sheath. The
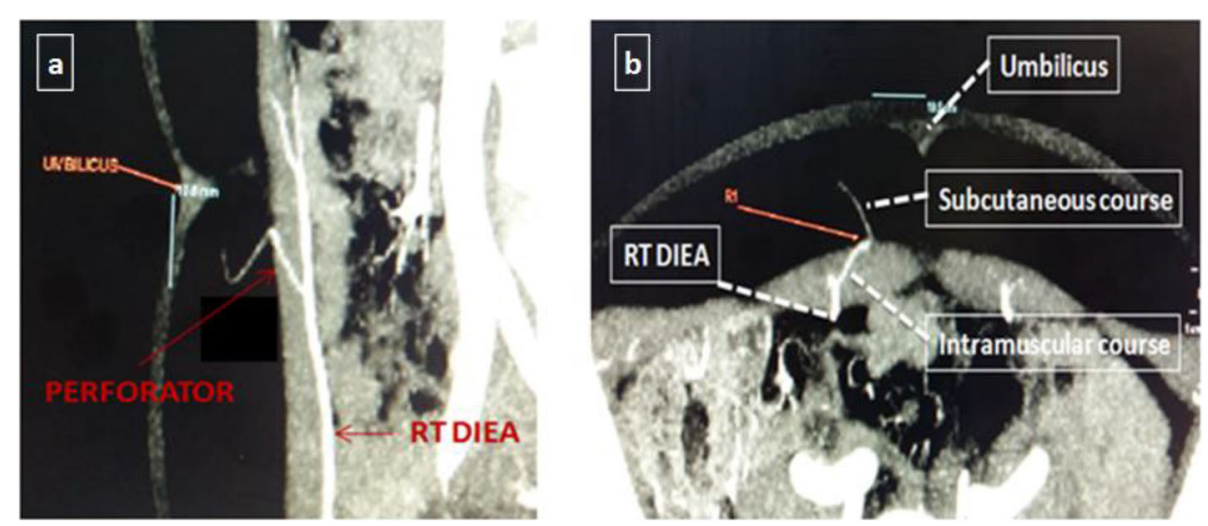

Fig. 3 Sagittal (a) and axial (b) thin maximum intensity projections of the anterior abdominal wall showing the course of the intramuscular segment and site of the piercing of the rectus by a dominant perforator in relation to the umbilicus 

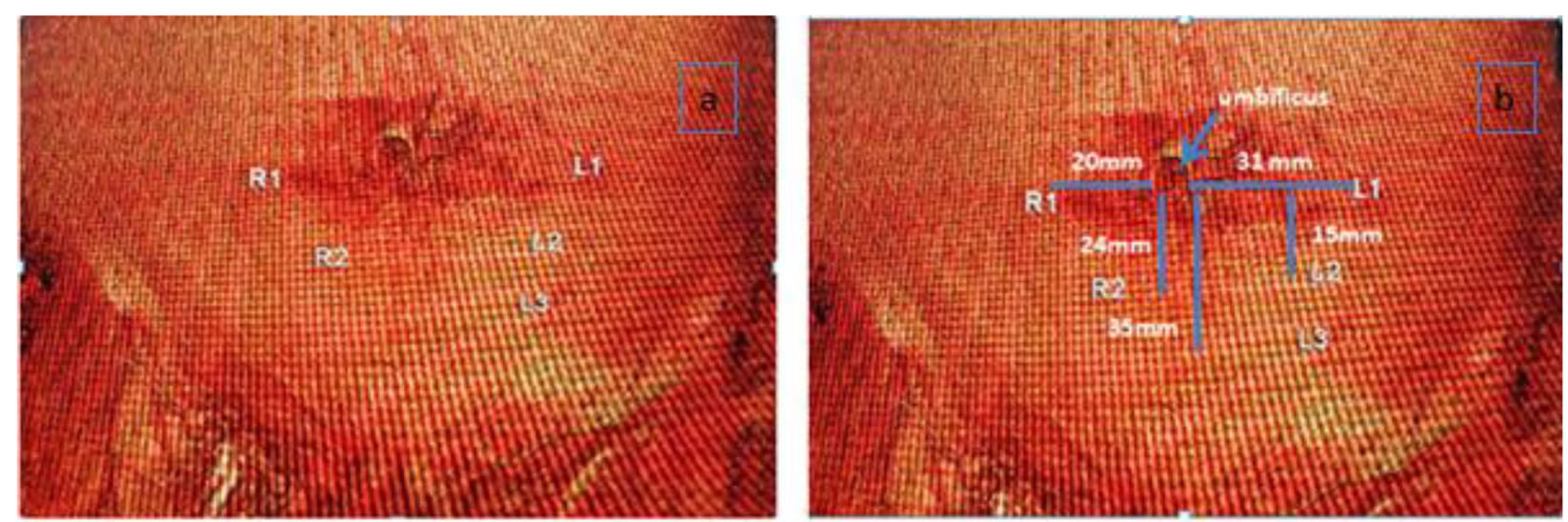

Fig. 4 Coronal volume-rendered (VR) images of the anterior abdominal wall showing the mapping of the deep inferior epigastric artery perforators (a) and their site in relation to the umbilicus (b)

larger the caliber of the perforator the better. It was agreed that a caliber more than $1 \mathrm{~mm}$ is preferable.

3) Length of the intramuscular segment was evaluated on the axial and sagittal MIP images. A short length of less than $3 \mathrm{~cm}$ was needed.

4) Course: a tortuous or oblique course was less desirable to avoid excessive muscle damage from dissection. The more perpendicular the course of the perforator the more preferable.

\section{Statistical analysis}

Statistical package for Social Sciences for Windows 15.0 (SPSS Inc., Chicago, IL, USA) was utilized to perform the statistical analysis.

The sensitivity, specificity, accuracy, positive predictive value, and negative predictive values for detection of the site, caliber, length, and orientation of the intramuscular course of each perforator by P-CTA and CDUS were compared to the surgical results that were considered as
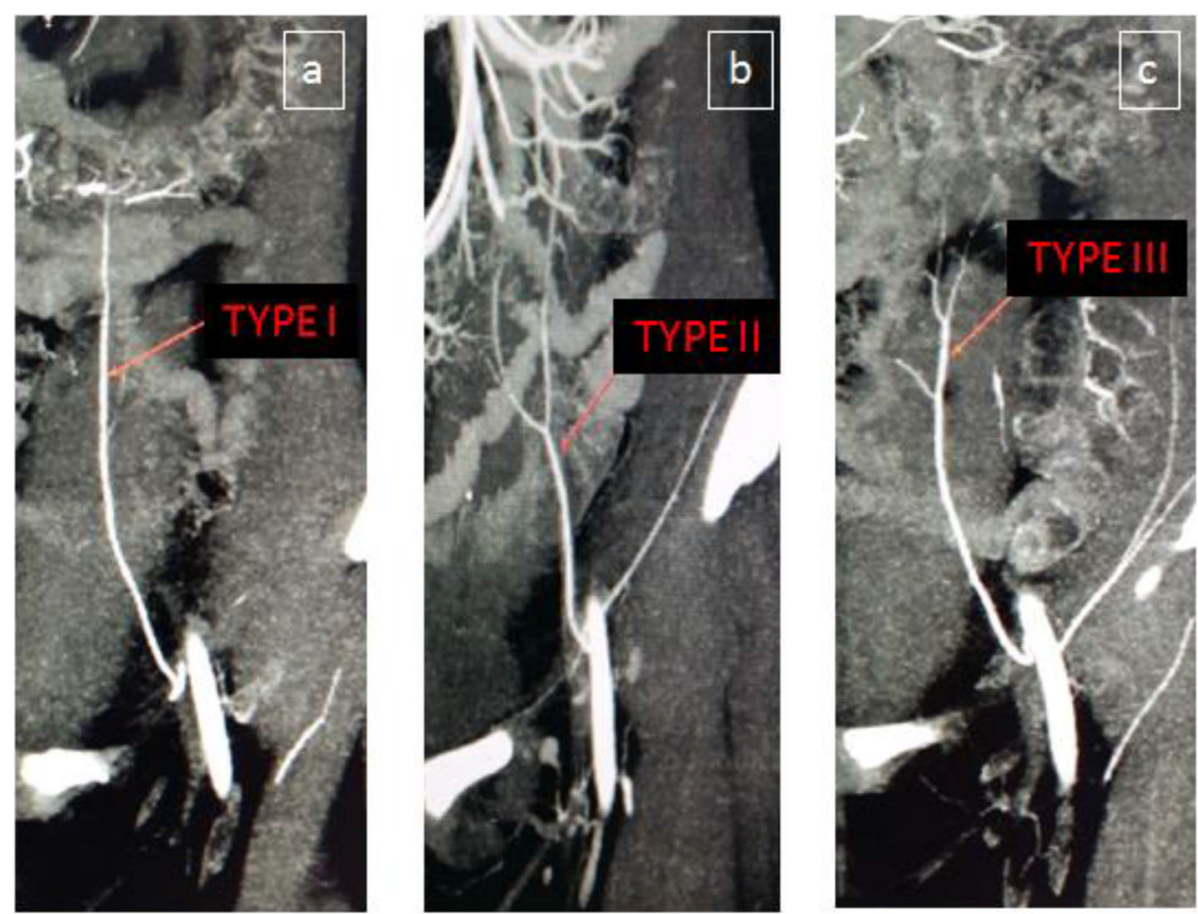

Fig. 5 Coronal maximum intensity projections (MIP) showing the three different branching patterns of the deep inferior epigastric artery according to the Moon and Taylor classification as follows: a a single trunk, $\mathbf{b}$ bifurcating into medial and lateral branches, or $\mathbf{c}$ subdividing into three or more branches 

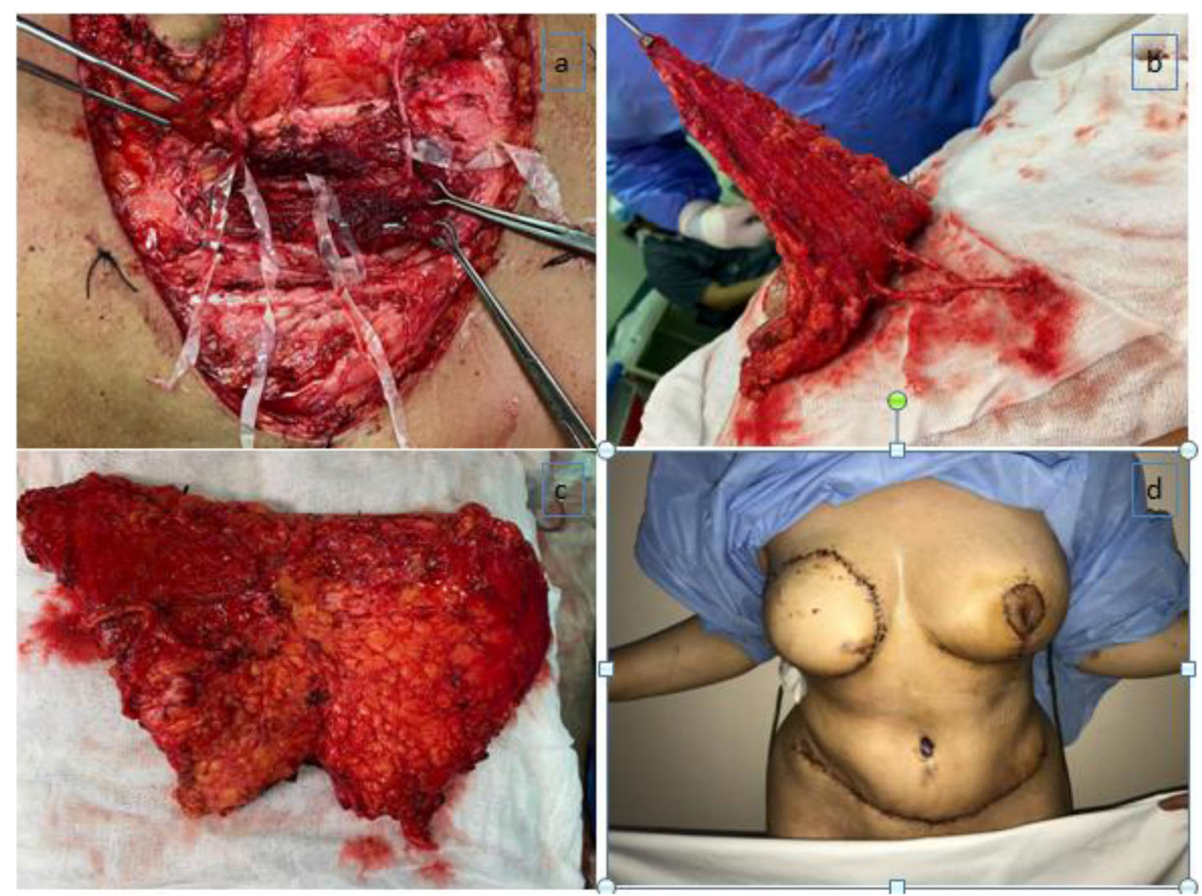

Fig. 6 The intra-operative perforator flap methodology: a elliptical incision through the lower abdominal adipocutaneous tissue to delineate the origins of the perforators from the deep inferior epigastric artery, $\mathbf{b}, \mathbf{c}$ dominant perforator artery identified and dissected with mobilization of the flap (d) reconstructed breast after anastomosis of the mobilized flap with the vessels at the recipient site. In addition, there is left nipple reconstruction noted

a gold standard. A $p$ value of less than 0.05 was considered statistically significant.

Weighted kappa statistics were used to calculate agreement between P-CTA and CDUS. The strength of agreement was interpreted according to the Landis and Koch guidelines as follows: 0: poor, 0.01-0.20: slight, 0.210.40: fair, 0.41-0.60: moderate, 0.61-0.80: substantial, and 0.81-1.00: almost perfect.

\section{Results}

A dominant perforator was detected by CDUS in 41 out of 42 patients and by P-CTA in 42 out of 42 patients. Surgical results identified a total of 96 dominant perforator vessels. There were statistically significant differences $(P=0.0025)$ between CDUS and P-CTA in the detection of the number of perforators. P-CTA proved to be more accurate than CDUS in detecting the number of dominant perforators being able to demonstrate 93 dominant perforators compared to 83 only by CDUS.

The sensitivity, specificity, accuracy, positive predictive value (PPV), and negative predictive value (NPV) of $\mathrm{P}-$ CTA in comparison to surgical findings in localization of the site of the perforator in relation to the umbilicus, caliber of perforator as it pierces the rectus abdominis, and length and course of the intramuscular segment of the perforator are demonstrated in Table 1 . We found significant differences between P-CTA and surgical findings in measuring the caliber of dominant perforators $(P=0.002)$. CDUS was more accurate than P-CTA in measuring the caliber of the dominant perforator, and P-CTA sensitivity and specificity were 81 and $77 \%$, respectively, compared to 94 and $96 \%$, respectively, for CDUS. We did not find statistically significant differences between P-CTA and surgical findings for the evaluation of the site, length, and course of the intramuscular segment of the dominant perforator artery $(P=0.34)$.

The sensitivity, specificity, accuracy, positive predictive value (PPV), and negative predictive value (NPV) of CDUS in comparison to surgical findings in localization of the site of the perforator in relation to the umbilicus, the caliber of perforator as it pierces the rectus

Table 1 Summarized the accuracy of P-CTA in the detection of the different parameters of each perforator; site in relation to the umbilicus, caliber of perforator as it pierces the rectus abdominis, and length and course of the intramuscular segment of the perforator $(p<0.05)$

\begin{tabular}{llllll}
\hline & Sensitivity & Specificity & Accuracy & PPV & NPV \\
\hline Site & 95 & 96 & 97 & 98 & 99 \\
Caliber & 81 & 77 & 85 & 86 & 87 \\
Length & 91 & 94 & 93 & 93 & 97 \\
Course & 96 & 97 & 98 & 98 & 99 \\
\hline
\end{tabular}


Table 2 Summarized the accuracy of CDUS in the detection of the different parameters of each perforator; site in relation to the umbilicus, caliber of perforator as it pierces the rectus abdominis, and length and course of the intramuscular segment of the perforator $(p<0.05)$

\begin{tabular}{llllll}
\hline & Sensitivity & Specificity & Accuracy & PPV & NPV \\
\hline Site & 86 & 78 & 89 & 76 & 82 \\
Caliber & 94 & 96 & 97 & 96 & 99 \\
Length & 94 & 96 & 97 & 96 & 98 \\
Course & 82 & 86 & 84 & 83 & 87 \\
\hline
\end{tabular}

abdominis, and the length and course of the intramuscular segment of the perforator are demonstrated in Table 2. We found significant differences between CDUS and surgical findings in the detection of the course of the intramuscular segment of the perforator $(P=0.0028)$. P-CTA was more accurate in the detection of the course of the perforator, and CDUS sensitivity and specificity were 82 and $86 \%$, respectively, compared to 96 and 97\%, respectively, for P-CTA. We did not find statistically significant differences between CDUS and surgical findings for the evaluation of the site, caliber, and length of the intramuscular course of the dominant perforator artery $(P=0.40)$.

We found only fair agreement between P-CTA and CDUS in the measurement of the caliber of the dominant perforator artery as it pierces the rectus abdominis fascia as well as in the detection of the course intramuscular segment of the perforator (Table 3).

There was no significant difference as regards the average time required for examination and vascular mapping of the perforators for P-CTA and CDUS which were on average $30 \mathrm{~min}$ and $27 \mathrm{~min}$, respectively. However, there was a significant difference in the acquisition time between CDUS and P-CTA being on average 28 min and $40 \mathrm{~s}$, respectively.

\section{Discussion}

Breast reconstruction with the use of perforator flaps has increased the demand for autologous breast reconstruction over artificial breast reconstruction in the last decade. Recently, modified techniques in perforator flaps

Table 3 Agreement between P-CTA and CDUS in the detection of the deep inferior epigastric artery perforator; site in relation to the umbilicus, caliber of perforator as it pierces the rectus abdominis, and course of the intramuscular segment

\begin{tabular}{llll}
\hline & $\%$ agreement & $k$ & Interpretation \\
\hline Site & 95 & 0.915 & Almost perfect \\
Caliber & 60 & 0.310 & fair \\
Length & 79 & 0.550 & moderate \\
Course & 62 & 0.350 & fair \\
\hline
\end{tabular}

have refined the transfer techniques, thus preserving the innervation and vascularity of the donor's muscle and minimizing donor's site morbidity. This allowed yielding a cutaneous flap with improved vascularity [7].

Recently, DIEAP flap procedure is considered as the ideal surgical procedure for autologous breast reconstruction in cases following mastectomy and therapeutic radiation therapy for breast cancer [9]. This was accompanied by a continuous increasing request for accurate preoperative assessment by different diagnostic modalities [4].

Different diagnostic imaging modalities are available for pre-operative mapping of perforators including color Duplex ultrasonography (CDUS), perforator computed tomographic angiography (P-CTA) with MDCT, and magnetic resonance angiography (MRA). In our study, we compared the pre-operative imaging results of CDUS and P-CTA.

Color Doppler ultrasonography (CDUS) is the most commonly utilized modality for preoperative vascular mapping, compared with other diagnostic tools due to its portability, reasonable cost, and its ability to provide hemodynamic information, such as flow velocity and pulsatility as well as the lack of ionizing radiation. However, it has some limitations being operator dependent, time-consuming, low reproducibility, and steep learning curve [2].

In our study, we disagreed with Rozen et al. [10] which stated that CDUS could not detect or identify any perforators in his study. In our study, CDUS showed relatively high sensitivity and specificity (86 and $78 \%$, respectively) in detecting the site of the perforators in spite of being relatively lower in comparison to the P-CTA results which were 95 and $96 \%$, respectively. This difference is due to the relatively low PPV and NPV of doppler (76 and $82 \%$, respectively) in comparison to P-CTA which was attributed to the low sensitivity of CDUS to the perforators in deeper tissues especially in obese patients and because of the intermittent image capture.

Cine et al. [4] found that P-CTA showed a slight advantage over CDUS in the evaluation of the intramuscular course of perforator vessels which is in an agreement with our study which showed a significant difference in the evaluation of the intramuscular course of the perforators being higher with P-CTA. This is due to the inability of CDUS to provide 3D structural images of the perforators.

P-CTA with MDCT has the advantage of displaying three-dimensional images of the perforator vessels providing detailed structural information of their caliber and course, as well as, being easily reproducible with short scan acquisition time and low-operator dependability. Nevertheless, exposure to ionizing radiation and use of nephrotoxic contrast agents remain as major disadvantages of this modality [11]. 
In our study, we found that P-CTA accurately identified the location of the perforator with high sensitivity and specificity of 95 and $96 \%$, respectively, which is in agreement with Alonso-Burgos et al. [3]. However, it showed relatively low sensitivity and specificity in comparison to the CDUS and surgical results in measuring the caliber of the perforators. This is because in P-CTA, we measure the intraluminal opacified diameter while in surgery, the external wall to wall diameter and sometimes the surrounding adventitia are included in the perforator diameter. This is in disagreement with Masia et al. [5] study that showed no significant difference in perforator diameter measurement between P-CTA and surgery.

In our study, radiation measurement was not considered a priority which we acknowledge as a limitation of this study. However, Cina et al. [4] and Rozen et al. [11] revealed that X-ray exposure was consistent with values reported in routine pelvic CT scans.

Overall, our study demonstrated that the reconstructed multiple planar, MIP, and VRT images of perforators and the surrounding structures facilitated easier surgical interpretation and significantly reduced the operative time with better post-operative aesthetic results of the reconstructed breast mounds.

\section{Conclusion}

In conclusion, the pre-operative mapping of perforators by CDUS and P-CTA proved to be effective and complementary to each other in the localization and vascular mapping of deep inferior epigastric artery perforators, thus greatly helping vascular surgeons in performing this surgical procedure.

\section{Recommendations}

We advise further evaluation of magnetic resonance angiography as an alternative to P-CTA to overcome the problem of exposure to ionizing radiation encountered during P-CTA.

\section{Abbreviations}

2D: Two-dimensional; ADS: Acoustic Doppler sonography; CDUS: Color Doppler ultrasonography; DIEAP: Deep inferior epigastric artery perforator flap; MDCT: Multidetector computed tomography; MIP: Maximum intensity projection; MPR: Multiplanar reformat; NPV: Negative predictive value; PCTA: Perforator computed tomographic angiography; PPV: Positive predictive value; TRAM: Transverse rectus abdominis myocutaneous pedicle flap: VR: Volume-rendered reformat

\section{Acknowledgements}

Not applicable

\section{Authors' contributions}

All authors have read and approved the manuscript. Hazem Hamed Soliman (HHS), Mohamed F Abozeid (MFA), Maged Abdelrahman Moustafa (MAM). HHS, MFA, and MAM designed the research. HHS, MFA, and MAM performed the research. HHS analyzed the data. HHS and MAM wrote the paper.

\section{Availability of data and materials}

The datasets used and/or analyzed during the current study are available from the corresponding author on reasonable request.

Ethics approval and consent to participate

This study was approved by the research ethics committee of the Radiology Department of the Faculty of Medicine at Cairo University on April 08, 2018; reference number of approval: 245-2018. All patients included in this research gave written informed consent to participate in this study.

\section{Consent for publication}

All patients included in this research gave written informed consent to publish the data contained within this study.

\section{Competing interests}

The authors declare that they have no competing interests.

\section{Author details}

${ }^{1}$ Diagnostic and Intervention Radiology Department, Cairo University Hospitals, Kasr Al-Ainy, El-Manial, Cairo 11956, Egypt. ${ }^{2}$ Surgery Department, Cairo University Hospitals, Kasr Al-Ainy, Cairo, Egypt.

Received: 20 December 2019 Accepted: 2 June 2020

Published online: 09 June 2020

\section{References}

1. Ibrahim A.S., Khaled H.M., Mikhail N.N., Baraka H., \& Kamel H. (2014). Cancer incidence in Egypt: results of the national population-based cancer registry program. Journal of cancer epidemiology, 18 pages. https://doi.org/10.1155/ 2014/437971

2. Karunanithy N, Rose V, Lim AK, Mitchell A (2011) CT angiography of inferior epigastric and gluteal perforating arteries before free flap breast reconstruction. Radiographics 31(5):1307-1319

3. Alonso-Burgos A, Garcia-Tutor E, Bastarrika G, Cano D, Martinez-Cuesta A, Pina $L J$ (2006) Preoperative planning of deep inferior epigastric artery perforator flap reconstruction with multislice-CT angiography: imaging findings and initial experience. J Plast Reconstr Aesthet Surg 59(6):585-593

4. Cina A, Salgarello M, Barone-Adesi L, Rinaldi P, Bonomo L (2010) Planning breast reconstruction with deep inferior epigastric artery perforating vessels: multidetector CT angiography versus color Doppler US. Radiology 255(3): 979-987

5. Masia J, Clavero JA, Larranaga JR, Alomar X, Pons G, Serret P (2006) Multidetector-row computed tomography in the planning of abdominal perforator flaps. J Plast Reconstr Aesthet Surg 59(6):594-599

6. Phillips TJ, Stella DL, Rozen WM, Ashton M, Taylor GI (2008) Abdominal wall CT angiography: a detailed account of a newly established preoperative imaging technique. Radiology 249(1):32-44

7. Ono S, Hayashi H, Ohi H, Ogawa R (2017) Imaging studies for preoperative planning of perforator flaps: an overview. Clin Plast Surg 44(1):21-30

8. Rosson GD, Williams CG, Fishman EK, Singh NK (2007) 3D CT angiography of abdominal wall vascular perforators to plan DIEAP flaps. Microsurgery: Official Journal of the International Microsurgical Society and the European Federation of Societies for Microsurgery 27(8):641-646

9. DE, C. Greef. (2005). Breast reconstruction by DIEP free flap: about 100 cases. In Annales de chirurgie plastique et esthetique (Vol. 50: p. 56-61).

10. Rozen WM, Phillips TJ, Ashton MW, Stella DL, Gibson RN, Taylor Gl (2008) Preoperative imaging for DIEA perforator flaps: a comparative study of computed tomographic angiography and Doppler ultrasound. Plast Reconstr Surg 121(1):9-16

11. Rozen WM, Anavekar NS, Ashton MW, Stella DL, Grinsell D, Bloom RJ, Taylor Gl (2008) Does the preoperative imaging of perforators with CT angiography improve operative outcomes in breast reconstruction? Microsurgery: Official Journal of the International Microsurgical Society and the European Federation of Societies for Microsurgery 28(7):516-523

\section{Publisher's Note}

Springer Nature remains neutral with regard to jurisdictional claims in published maps and institutional affiliations. 